\title{
Los dilemas y perspectivas de futuro del docente becario de maestrías en educación
}

\author{
LUIS SIME POMA* \\ Pontificia Universidad Católica del Perú \\ Recibido el 17-01-2017, primera evaluación el 27-02-2017; \\ segunda evaluación el 09-03-2017; aceptación el 09-05-2017
}

\section{ReSUMeN}

Son diversos los programas de becas para docentes de educación escolar pública promovidos en América Latina. En el Perú, dicha política se gestionó desde Pronabec ofertando becas de maestría a docentes de diversas regiones del país, constituyendo una política inédita de formación continua durante 2014-2015. El presente estudio se propone: analizar los dilemas enfrentados por los docentes al postular a dicha beca; $y$, explorar las perspectivas de futuro profesional de los becarios. La metodología cualitativa implicó aplicar un cuestionario con respuestas abiertas a veinte becarios. Se logró analizar cuatro tipos de dilemas - maestría versus familia; maestría versus escuela; maestría versus otro estudio; maestría versus no migrar- y ocho escenarios futuros en torno al campo profesional, académico y sindical-político.

Palabras clave: becas, estudiantes de posgrado; desarrollo profesional, profesores

Dilemmas and future perspectives of teachers in master fellowship program in education

\section{Abstract}

Different fellowships programs to public school teachers have been developed in Latin-American. In Perú, this new program has been managed by Pronabec financing fellowships for teachers of different regions during 2014-2015. This study is addressed to analyze the teachers' dilemmas faced when they applied; and, explore the future professional perspectives of this kind of teacher. The qualitative

Ph D en Ciencias Sociales de la Radboud University (Nijmegen-Holanda), Magíster en Ciencias de la Educación de la Pontificia Universidad Católica de Chile y profesor principal del Departamento de Educación de la Pontificia Universidad Católica del Perú (PUCP). Actualmente es director del Doctorado en Ciencias de la Educación. Contacto: 1sime@pucp.pe 
method implies a documental analysis and survey with open answers to 20 teachers. Four types of dilemmas were identified-master versus family, master versus school, master versus another study, master versus no migration-, and eight possible futures scenarios related to the professional, academic, and unionpolitical fields.

Keywords: fellowships; postgraduate student; professional development; teachers

\section{Dilemas e perspectivas futuras da professores bolsistas da mestrado em educação}

\section{Resumo}

São diversos os programas de bolsas de estudo para professores de escolas públicas promovidas na América Latina. No Peru, a programa é administrado desde Pronabec oferecendo bolsas de estudo para professores de várias regióes do país durante 2014-2015, constituindo uma política sem precedentes de formação contínua. Este estudo tem como objetivo: analisar os dilemas enfrentados pelos professores para solicitar a bolsa de estudos; e explorar perspectivas de carreira futura dos professores. A metodologia qualitativa envolveu uma análise de documentos e um questionário de respostas abertas para 20 professores. Foi possível analisar quatro tipos de dilemas -mestrado contra a família; mestrado contra a escola; mestrado contra outro estudo; mestre contra não migrar- $\mathrm{y}$ oito cenários futuros em o campo profissional, acadêmico e sindical- político.

Palavras-chave: bolsa de estudo, estudantes de pós-graduação, desenvolvimento profissional, professor

\section{INTRODUCCIÓN}

La formación continua de los profesores de educación básica ha ido emergiendo como un campo de estudio específico motivado en gran medida por el incremento y diversificación de las políticas para mejorar su desarrollo profesional como unos de los factores claves de la calidad educativa (OECD, 2014). Como parte de dichas políticas, en diversos países de América Latina (AL) se han impulsado en esta última década diferentes programas públicos para becar a los docentes a fin de realizar estudios de posgrado en educación.

Son diversos los programas de becas promovidos tanto desde los gobiernos nacionales como subnacionales en AL; así, en Colombia, el Ministerio de Educación desarrolla el Programa "Becas de Excelencia Docente", para estudios de maestría de dos ańos en universidades públicas o privadas acreditadas 
en el país, y que aspira a una cobertura de 7557 becarios para 2015-2016 (Mineducación, 2016). En la capital, la Alcaldía Mayor de Bogotá (2012), se trazó en su plan 2012-106, que el 30\% de los docentes y/o directivos docentes de ese ámbito territorial tuvieran una formación de excelencia en doctorados, maestrías y especializaciones. Esta política ha sido desarrollada esperando beneficiar a más de 8000 docentes en base a becas que cubren el 70\% de los gastos académicos (Red Académica Bogotá, s.f.).

En Brasil, la meta del Plan Nacional de Educación prevé que al 2014 la mitad de sus docentes tendrán estudios de posgrado y el crecimiento de profesores en aula con posgrado ha evolucionado del $25,2 \%$ en 2008 al 31,1\% en 2014 (Foreque, 2015). Entre las iniciativas de los Estados Federales destaca la Secretaria da Educação do Estado de São Paulo (2015), que ofrece desde 2012 ayudas financieras para los docentes de su red pública de escuelas, tanto para estudios de maestría como de doctorado.

A su vez, el Ministerio de Educación de Chile también ha ofertado becas de magíster para docentes que hayan participado en procesos de evaluación de desempeño y se encuentren ejerciendo en escuelas subvencionadas por el Estado, para ser cursados en universidades con maestrías acreditadas y por un plazo de hasta dos años; siendo 68 los admitidos para el periodo 2014-2015 de 291 postulaciones (Conicyt, 2014). Igualmente, han sido ofertadas en 2013, 60 becas para maestrías en el extranjero (Conicyt, 2013).

Estas políticas de estímulos a la formación de posgrado de los profesores de educación básica exige desde el lado de la comunidad investigadora el configurar una subárea de estudio que requiere de perspectivas de análisis para lograr una mayor singularización de las experiencias y percepciones de los profesores que continúan esa formación, especialmente a través de las maestrías en educación, siendo estas una de las más numerosas en AL (Rama, 2004; Gusmão, 2010).

Las investigaciones sobre los docentes de educación básica con formación de posgrado han venido enriqueciéndose con los aportes que analizan los antecedentes académicos de los profesores para medir la proporción de los docentes con maestrías y otros grados. Así, Ingersoll (2011) compara esos porcentajes, respecto al conjunto de la población docente, en cinco países asiáticos y Estados Unidos. El análisis de esa proporcionalidad viene también estimulada por el impacto de las evaluaciones internacionales, como PISA, en la cual se identifica que en los países con mejores resultados, sus docentes cuentan con estudios de posgrado (Sahlberg, 2010). También se ha utilizado la variable de antecedentes académicos de los docentes para correlacionarlos con otras variables, como la deserción docente (Ingersoll, Merill \& May, 2014). 
Otros autores han indagado el "efecto maestría": la incidencia que pueden ejercer los docentes con maestría en el rendimiento de los estudiantes (Tobón, Valencia, Ríos \& Fredy, 2008; Harris \& Sass, 2007; Darling-Hammond, 2000; Gandhi, 2006).

Más específicamente, las investigaciones sobre docentes de educación básica becarios de programas públicos para estudiar maestrías en AL, son muy escasas según la exploración realizada en las indexadoras de revistas como Redalyc y Scielo, a través de descriptores como "becas docentes"/ "posgrados educación"/ "becas posgrado", tanto en español como en portugués.

En este trabajo proponemos contribuir a ese objeto específico de estudio con acercamientos que visibilicen más al docente como un sujeto que pone en juego sus propias subjetividades desde el inicio de su experiencia de formación de posgrado. En ese sentido, este acercamiento complementa la visión del docente como producto final de las maestrías, más estudiado por los enfoques aludidos anteriormente. En esa misma línea de contribución, situamos el estudio sobre las orientaciones hacia el futuro profesional que los docentes vislumbran. Desde autores como Nuttin - parafraseado por Martínez (2006, p. 113) — "la conducta posee una dimensión temporal, así, gran parte de nuestros actos se pueden entender en función a las metas, proyectos o deseos que tenemos para el futuro". Retomando a Zaleski, Martínez (2006, p. 113) señala que: "El futuro entendido como un espacio de planeamiento y realización de metas, tiene en ese sentido un poder motivacional, no obstante, el futuro puede ser asimismo un espacio de aspectos negativos e incertidumbre."

Desde el caso peruano, las políticas de formación continua para los docentes de educación básica han sido organizadas como programas de capacitación que no derivaban en la obtención de un grado académico de Maestría (Cuenca \& Stojnic, 2008; Sánchez, 2006). Sin embargo, desde hace tres años, el Programa Nacional de Becas y Crédito Educativo (Pronabec), adscrito al Ministerio de Educación del Perú, gestionó fondos públicos para ofertar becas integrales de maestría a docentes de diversas regiones del país, constituyendo una política inédita de formación continua en el país.

El presente estudio ha tenido como objetivos: analizar los dilemas enfrentados por los docentes al postular a dicha beca; y, explorar las perspectivas de futuro profesional de los docentes becarios. 


\section{LOS DILEMAS PROFESIONALES Y LAS PERSPECTIVAS DE FUTURO PROFESIONAL}

El concepto de dilemas profesionales busca enriquecer el campo de estudio sobre las carreras profesionales enfatizando en aquellos momentos significativos que atraviesan los profesionales y que a pesar de su uso en ciertos trabajos este no ha sido suficientemente conceptualizado (Tharenou, 2015). Partimos por diferenciar dos significaciones del concepto de dilema profesional. Siguiendo a Schön (1992), distinguimos los "dilemas de la práctica" para revelar situaciones emergentes de las prácticas profesionales cotidianas ante las cuales el profesional debe tomar decisiones entre varias posibilidades para continuar con su labor dependiendo también de la profesión en cuestión (Cherry, 2014). Fransson y Grannäs (2013) han realizado un esfuerzo conceptual para caracterizar los contextos educativos como espacios esencialmente dilemáticos donde acontecen dilemas con diversos niveles de complejidad y que exigen toma de posiciones y de negociaciones y, al igual que lo mencionado por Minner y Beane (1983), contienen tensiones entre las percepciones de los sujetos implicados en las situaciones, así como, pueden subyacer conflictos entre macro tendencias institucionales y las micro experiencias influidas por factores culturales diferentes (Sheng-nan, p. Da-ming, 2015).

También existen otros tipos de dilemas que revelan situaciones que demandan decisiones entre dos o más opciones con impactos diferentes en la trayectoria del profesional y que son los "dilemas de carrera" (Santos, 2004), aplicable para decisiones como el asumir o no un nuevo trabajo o una responsabilidad diferente en la misma institución. En ese sentido, las trayectorias profesionales pueden ser consideradas como construcciones dilemáticas: los profesionales tendemos a enfrentar múltiples dilemas de carrera (Pittman, 2000). Quadrado y Rocha-de-Oliveira (2015), afirman que en un momento dado los profesionales tienen diversas alternativas en su carrera, representando posibles trayectorias a ser asumidas y que los estudios deben contribuir al análisis de esas elecciones en esas coyunturas de incertidumbre.

Una tipología de dilemas de carrera es la propuesta por Sime (2008) que diferencia 5 tipos. Los vocacionales son aquellos momentos en los que el sujeto debe decidir entre reafirmar su vocación por su profesión o retirarse definitivamente; los de campo, cuando se trata de reubicarse en otro ámbito dentro del propio campo profesional; lo denominados geográficos son los que implican la decisión de migrar hacia otra ciudad o país; lo institucionales refieren a la reubicación laboral dentro de la misma institución, y, finalmente, los de especialización que atańe a las decisiones sobre continuar estudios posteriores a su formación inicial. El autor sostiene la posibilidad de diferentes interrelaciones 
entre ellos, así, por ejemplo, al enfrentar el dilema de especialización también se podría estar enfrentando el dilema geográfico, en la medida que la decisión por continuar estudios signifique una decisión de migrar.

Una delimitación al concepto de dilemas de carrera es que estos surgen del propio ámbito profesional y no deben ser confundidos con otros que ocurren en el ámbito más personal, como decidir o no en contraer matrimonio o divorciarse; aunque existan influencias mutuas. En suma, los dilemas de carrera se conectan con una visión retrospectiva de las trayectorias profesionales estudiadas estas últimas como parte del campo de estudios sobre "career paths" o "career trayectories" (Cochran, Carter , p. Dorsey, 2003; Latifoglu, 2016; Johnston-Anderson, 2016; Eckert, Ulmer, Khachatryan , p. Ledesma, 2016).

La conceptualización de los dilemas de carrera en el campo docente está vinculado a los estudios sobre la vida y trayectoria profesional de los profesores desde una perspectiva subjetiva. Rinke (2008) señala que un aspecto común de estos estudios es intentar comprender cómo los docentes construyen y entienden su propia vida profesional. Uno de los enfoques más comprehensivos sobre las biografías profesionales de los docentes es el que aporta Kelchtermans (1993), para ir más allá de la trayectoria formal del docente y reconocer la presencia de incidentes críticos, fases críticas y personas críticas - de mayor influencia - en esa trayectoria. Para este autor, la comprensión del yo profesional requiere de una perspectiva retrospectiva y a la vez prospectiva. La primera implica explorar sobre cómo los docentes se conciben a sí mismos como docentes no de una manera estática sino dinámica a lo largo del tiempo. Un aspecto que el autor resalta en esa reconstrucción son los cambios en la motivación laboral del docente y, aunque no utiliza el concepto de dilema de carrera, lo esboza, al señalar la relación entre su motivación y el dilema de permanecer o salir de su trabajo docente. La prospectiva del docente es concebida por este autor como las expectativas y sentimientos que el docente presenta sobre su futuro desarrollo profesional donde puede anticipar problemas o limitaciones para continuar en la misma labor. Esta parte, sobre las perspectivas de futuro de los docentes, es limitada no solo en el texto de Kelchtermans, sino también en otros involucrados en el estudio de las trayectorias profesionales docentes.

El interés por reconocer la dimensión prospectiva de la profesión docente empieza a ser considerada en algunas encuestas nacionales a profesores de educación básica en América Latina. En ellas se incluye al menos una pregunta sobre cuál es la perspectiva laboral que los docentes avizoran en los próximos años, como la aplicada en el Brasil (Andrade, p. Fraga, 2010), en el Perú 
(Minedu-CNE, 2015) y en los Estados Unidos (The MetLife, 2013). Las indagaciones sobre las perspectivas laborales futuras de los docentes pueden constituir un referente para un diálogo entre las políticas docentes y sus aspiraciones profesionales y, en el campo de la investigación, puede aportar a reconocer cómo los docentes construyen su sentido de continuidad y discontinuidad en sus carreras (van Rijswijk, Akkerman, Schaap, van Tartwijk, 2016).

Además de la perspectiva subjetiva, el enfoque de agencia profesional (Eteläpelto, Vähäsantanen \& Hökkä, 2015), nutrido desde aportes multidisciplinarios (Zavala y Castańeda, 2014), permite conceptualizar los niveles de protagonismo de los sujetos profesionales sobre sus propias vidas e integrar aspectos abordados en los párrafos previos. Por un lado, esos niveles de protagonismo son tensionados en momentos de elecciones fuertes, como los dilemas de carrera, y cargados subjetivamente por sus orientaciones laborales de futuro. Por otro lado, la agencia que desarrollan los sujetos implica un aprendizaje biográfico: “...agency is not simply concerned with the ways in which we engage with our contexts-for-action but rather has to do with the capacity to shape our responsiveness to the situations we encounter in our lives. It is the latter insight which allowed us to articulate a relationship between agency and biographical learning...” (Biesta \& Tedder, 2007, p. 146).

\section{La beca docente de Pronabec}

Desde 2013, el Pronabec abre una línea de financiamiento público de becas docentes para cursos de especialización y de maestría. La primera ha beneficiado a más de 10761 docentes de educación básica regular (EBR) y, la segunda, a 553 profesores nombrados (no contratados) (Pronabec, 2015), con una inversión de 30 millones de soles (El Peruano, 2014), en un universo de docentes del sector público que asciende aproximadamente a 317000 docentes (Minedu, 2014). Como referencia contextual, el porcentaje de directores y docentes de EBR con maestría es menor al $12 \%$ en cualquiera de los niveles educativos de enseñanza según la encuesta del Instituto Nacional de Estadística e Informática (Inei, 2014).

Una primera característica sobre la cobertura de este tipo de beca es su enfoque descentralizado: $73 \%$ de los docentes provienen de regiones del interior y $27 \%$ son de la capital. Una segunda es la proporcionalidad de género: $55 \%$ para mujeres y $45 \%$ para varones (Pronabec, 2015). Un tercer rasgo es la meritocracia en la selección de los becarios, los cuales pasaron por la evaluación de Pronabec y por las cuatro universidades privadas seleccionadas por ese organismo comprometidas para ofrecer los estudios de maestría en cuatro 
semestres académicos. De esta manera, de los 1495 postulantes fueron admitidos 607 docentes, el 40\% del total (Minedu-Pronabec, 2014).

Otra característica, es el tipo de compromisos exigidos a los docentes como la dedicación de tiempo completo durante un año y medio para culminar su formación; y el continuar por el doble del tiempo en su institución educativa luego de culminar los estudios. Un rasgo adicional de esta política es el financiamiento integral sin devolución, la no pérdida de la remuneración del docente durante el tiempo de sus estudios y una subvención para los gastos de mantenimiento. Sin duda, estas características convierten a esta beca en una oportunidad de formación privilegiada entre los profesionales que laboran en el Estado peruano. En la Encuesta Nacional Docente (Minedu-Cne, 2015), el mayor porcentaje de interés que manifiestan los docentes encuestados del sector público para continuar con su desarrollo profesional recae en estudiar una maestría o doctorado (23,5\%).

Una última característica es la orientación pedagógica y profesional de las maestrías ofrecidas por las universidades, predominando las didácticas hacia un área curricular específica (ciencias naturales, matemáticas) y otras con orientaciones más transversales (TIC, innovación curricular; evaluación de aprendizaje).

Tabla 1. Orientación temática de las maestrías para docentes becarios

\begin{tabular}{lc}
\hline \multicolumn{1}{c}{ Orientación } & Docentes becarios \\
\hline $\begin{array}{l}\text { Investigación e innovación curricular } \\
\begin{array}{l}\text { Didáctica de la enseñanza en matemáticas en educación } \\
\text { secundaria }\end{array}\end{array}$ & $163(29 \%)$ \\
$\begin{array}{l}\text { Aprendizaje por competencia } \\
\text { Didáctica de la enseñanza en matemáticas en educación primaria }\end{array}$ & $78(14 \%)$ \\
$\begin{array}{l}\text { Didáctica de la enseñanza en educación inicial } \\
\text { Didáctica de la enseñanza en ciencias naturales en educación } \\
\text { primaria }\end{array}$ & $48(8 \%)$ \\
$\begin{array}{l}\text { Innovación de las tecnologías de la información y comunicación } \\
\text { Didáctica de la enseñanza en ciencias naturales en educación } \\
\text { secundaria }\end{array}$ & $41(7 \%)$ \\
& $36(6 \%)$ \\
\hline
\end{tabular}

Fuente: Pronabec $(2014,2015)$. 


\section{Acercamiento metodológico}

La metodología cualitativa desarrollada implicó para los objetivos planteados - analizar los dilemas enfrentados por los docentes al postular a dicha beca y explorar las perspectivas de futuro profesional de los becarios- la aplicación de un mismo cuestionario con respuestas abiertas. Este instrumento fue respondido por veinte docentes (hombres y mujeres; de los diferentes niveles de la educación básica del sector público) becarios de Pronabec y procedentes de distintas regiones del país que cursaron sus estudios en una maestría con orientación curricular, con dedicación a tiempo completo en una de las universidades privadas en la capital del país (Lima), durante el periodo del 2014-2015. Otra característica de este grupo es su condición de profesores que la literatura los considera a mitad de su carrera (Strahan, 2016), es decir, no son docentes principiantes ni tampoco están próximos a jubilarse.

El cuestionario aplicado para la recolección de la información durante el último semestre de 2015, en el contexto de uno de los cursos de dicha maestría, incluye preguntas que gatillan la reconstrucción narrativa del pasado y, a la vez, la visión prospectiva del docente. Sobre lo primero, los docentes fueron motivados para evocar aquellos dilemas significativos que han enfrentado en su trayectoria profesional, es decir, situaciones en las cuales debían decidir entre dos o más alternativas. Se procesó cualitativamente las respuestas que referían a dilemas enfrentados al postular a la maestría estableciéndose categorías emergentes al análisis descriptivo para luego proceder a la selección de las citas textuales más ejemplares.

Con relación a la segunda, se le solicitaba al docente redactar aquellos logros que le interesaría alcanzar profesionalmente o en estudios dentro de los próximos 3, 6 y 10 años. Las respuestas fueron procesadas en dos fases. En una primera se definieron en un matriz los escenarios de futuro derivados del análisis de cada respuesta; luego se procedió a tipificar las perspectivas profesionales proyectados por los docentes.

\section{Resultados}

\subsection{Los dilemas de los docentes becarios}

La postulación a los estudios de maestría ha sido una decisión significativa en las trayectorias de los docentes becarios marcada por el afrontamiento de al menos cuatro tipos de dilemas: maestría versus familia; maestría versus escuela; maestría versus otro estudio; maestría versus no migrar. 
Sin duda, el dilema más reiterado por los docentes ha sido el primero, significando tensiones con la familia al momento de la decisión y la necesidad de reconstruir un equilibrio entre las demandas de los estudios con las demandas familiares. Estas últimas demandas se agudizan para los casos de docentes con hijos menores o con padres mayores. De esta manera, la decisión de postular e iniciar los estudios de maestría implicó un impacto inmediato en el ámbito familiar que es percibido como una ausencia crítica con alto costo emocional:

...mis hijos solos en la casa, quién los ayudaba en su tarea, sin embargo, decidí que sí podía y que mis pequeños podrían afrontar tal situación, hasta el momento es un poco complicado pero a pesar de todo sigo adelante. Si me volvieran a proponer otra beca no creo que volvería acertarla, mis hijos están creciendo y cambiando y necesitan más de mí. (D1)

Lo primero fue mi familia, especialmente mis hijas, si inicialmente estaba muy decidida de traerlas conmigo a Lima poco a poco esa idea se fue disipando por el horario de estudio de la maestría sobre todo y el costo de vida que implica permanecer en este lugar. Esto me afectó, me afecta emocionalmente porque ellas son el motor de mi vida y es muy doloroso tener que dejarlas. Este fue y es el dilema más fuerte y entristecedor. (D2)

Las implicancias familiares son muy grandes pues los hijos pequeños resienten tan largas ausencias. La principal afectada es mi hija de 7 años...(D3)

La decisión de aceptar la beca significó reacciones distintas por parte de los familiares. Así, hay docentes que contaron con un apoyo emocional positivo aun en circunstancias complejas, que derivó, además, en redistribuir los compromisos con el resto de la familia:

...gracias a Dios tuve el apoyo de mi familia que me alentó hacerlo, especialmente mi mamá, ella vive conmigo, tiene 83 años y se quedó sola cuando tome está decisión, más adelante al ver mi decisión mis hermanos decidieron apoyarme y uno de ellos visita ahora a mi mamá con más regularidad. (D6)

...mi esposa padecía con una enfermedad crónica y dejar toda mi rutina laboral era muy difícil. Estuve a punto de no firmar el compromiso. Conversé con mi esposa y ella me alentó. Finalmente decidí estudiar y a pesar de las dificultades sigo avanzando. (D4).

Hoy sigo estudiando y ellas [las hijas] también, ayudándonos unos a otros y muchas veces más independientes cada uno asume lo suyo, creo que fue una decisión acertada el cambiar el ritmo familiar y priorizar algunas cosas como los estudios adaptándose unos con mayor facilidad que otros y si se presentara otro contratiempo no dudaría en tomar decisiones adecuadas al tiempo y espacio. (D7) 
Una manera de racionalizar los costos de la decisión por aceptar la beca de estudios es la que plantea una docente, al asumirla como una experiencia referencial de estímulo para la formación posterior de sus hijos. Esto puede ser leído como una ganancia no solo en el presente y futuro profesional del docente sino también para los hijos en la medida que en el futuro ellos también apuesten por estudiar más como sus padres:

El apoyo que pueda brindar la familia es fundamental, la pareja y los hijos pueden hacer que se siga avanzando y los hijos terminan aprendiendo con el ejemplo más que con las palabras así es que recién se verán los frutos, a largo plazo. (D3)

En otros casos, la opción por la maestría tensionaba la relación con la escuela donde el docente ejercía al momento de postular. Desde un sentido más afectivo, el dejar la escuela está asociado a nostalgia y un cierto duelo sobre todo en aquellos con un largo tiempo en la misma escuela o que perciben haber aportado a la institución:

Fue una decisión difícil. Por cerca de quince años trabajé en dicho colegio... de mucho prestigio. Siempre pensé que allí me jubilaría. Había echado en mi escuela raíces... Un día le dije adiós y me vine para Lima con el corazón partido, pero lleno de fe y esperanza...(D11)

Asimismo, era la institución de todo lo que para mí significaba, los proyectos, mis estudiantes. Sé que salí en el mejor momento de mi carrera profesional y sobre todo porque las gestiones que hice empezaban a dar frutos y eso me llenó de satisfacción porque dejaba a la institución encaminada y con muchas acciones todavía por concretar que muy adecuadamente supo dirigir el director que me reemplazó. (D2)

Desde otra perspectiva, la idea de salir de la escuela para estudiar la maestría por un tiempo, está más bien legitimado por el mal momento de la institución: "En mi I.E. existían problemas a nivel de clima laboral y cambió de Dirección y pensar en un alejamiento pareció una buena idea." (D3).

En otros casos la opción por iniciar la maestría ha rivalizado con la opción por culminar otro tipo de estudio ya iniciado, sea de otra carrera u otros estudios de maestría.

Alternadamente con el ejercicio de mi profesión de docente fui estudiando la carrera de Derecho. Me decidí culminar el último año que me faltaba aprobar y graduarme, pero cuando...ya me había matriculado en el segundo semestre, me llaman de PRONABEC y me indican que había ganado la Beca docente y debía de trasladarme casi de inmediato a Lima lo que iba significar retrasarme 
otra vez en mi carrera de Derecho. En verdad fue un dilema muy grande porque era una muy buena opción para mi carrera de docente y mi ejercicio profesional pero significaba a la vez un retraso en mi otra carrera que de por sí ya me había dejado buenos ingresos económicos...(D9)

Respecto a la decisión en torno a la migración, esta es singularmente importante por las implicancias en las habilidades adaptativas del docente en su nuevo contexto. En la siguiente cita textual se advierte el reto que significaba asumir esa migración sobre todo para aquellos con escaso conocimiento de la nueva ciudad y considerando el lapso de tiempo para vivir en ella por más de un ańo.

...no me fue fácil decidir si debía presentarme o no, pues asumir ese nuevo reto implicaba entre otras cosas el mudarme de mi ciudad a otro lugar, el cual había visitado muy superficialmente... El dilema primeramente geográfico que tuve que enfrentar me permitió realizar un análisis reflexivo respecto de mi capacidad de adaptación a nuevos contextos, lo que considere una debilidad más que una fortaleza debido a que se me hizo difícil buscar los mecanismos para enfrentar un nuevo estilo de vida. (D10)

\subsection{Perspectivas de futuro profesional de los docentes becarios}

Las perspectivas que los docentes vislumbran sobre su futuro profesional giran en torno a ocho posibles escenarios que pueden ser agrupados en torno a tres campos: a) desarrollo profesional: aquí se subdivide en relación al ámbito de la educación básica, como asumir cargos de gestión, postular al ascenso en su carrera; mejorar su actual institución educativa; y al ámbito de la educación superior, como ingresar a la docencia-gestión en ese ámbito; y, al ámbito de crear una institución propia b) desarrollo académico: continuar con otros estudios; investigar y publicar c) desarrollo sindical-político: optar por un liderazgo sindical o político.

Con relación al primer campo de desarrollo profesional, 14 de los 20 docentes becarios prevén un futuro profesional vinculado a responsabilidades en la gestión de entidades de educación básica, sea a nivel de la escuela, en entidades intermedias y de alta gestión ("Después de ese tiempo y en adelante, me gustaría terminar mi carrera como funcionario de más alto nivel en el sector educación". D9).

Respecto al escenario para lograr un ascenso en su carrera magisterial, en seis de ellos, esta opción ha sido considerada en sus respuestas para acceder a la escala máxima, mayormente como aspiración después de los tres años. 
En cuanto a mejorar su actual institución educativa, llama la atención, tratándose de una maestría con orientación curricular, que solo cinco docentes incluyen como logros profesionales para alcanzar dentro de los siguientes tres años, el mejorar el currículo de su actual institución, el aprendizaje de sus alumnos o la formación contínua de sus pares. En solo uno de ellos, se explicita revertir la tesis en intervención: "Poner en ejecución a manera de proyecto la hipótesis planteada en mi investigación, existe correlación significativa entre la presencia de un gestor curricular y el logro de los aprendizajes" (D13).

Un poco más de la mitad de los profesores se perfilan trabajando en la universidad, mayormente en la enseńanza como catedráticos, en algunos casos aspirando a enseñar en universidades de prestigio.

Aproximadamente la cuarta parte de los veinte docentes becarios visualizan la creación o consolidación de una institución propia, sea una escuela o una ONG para realizar consultorías; en un caso se explicita que estas serían para el ministerio de educación y organismos internacionales; en otro, más especializado hacia la capacitación: "Tener un centro de capacitación y asesoramiento para docentes y directivos. Asociándome con otros colegas para formar una microempresa de capacitación. Presentando proyectos de capacitación a las municipalidades u otras instituciones" (D17); en otra respuesta, se alude a crear esta institución en asociación con los compañeros de la maestría cursada.

En cuanto al segundo campo de desarrollo académico, en trece de ellos, el escenario de continuación de estudios, se proyecta hacia un doctorado, en algunos fuera del país con una beca; mientras que cinco, expresan su intención por seguir otra maestría. En menor medida, cuatro desean hacer un estudio de especialización o una licenciatura; y tres se inclinan por estudiar idiomas. Solo uno se proyecta hasta estudios posdoctorales.

La mitad de los profesores revela como expectativa, investigar y publicar un libro o artículo. En ciertas respuestas la referencia es muy vaga; en otras, se precisa un tema: "Publicar mis producciones sobre educación física" (D14) y, en otras, se advierte una dispersión temática: "Realizar investigaciones respecto al currículo, aprendizaje, problemas de aprendizaje, interculturalidad y demás temas educativos para publicar artículos en revistas indexadas" (D2); "Escribir un libro sobre gestión del currículo en las escuelas públicas y otro sobre clima escolar y la tutoría" (D13). También hay algunos que enfatizan su experiencia profesional propia como la fuente para una publicación: "Escribiré un libro de mi trayectoria profesional" (D16); "Presentar un artículo académico en base alguna experiencia que haya realizado" (D11). Solo en una respuesta se hace referencia a publicar su tesis realizada en la maestría como docente becaria. 
En cuanto al campo del desarrollo sindical-político, solo tres docentes incluyeron el escenario del participación gremial-política, en su proyección para diez ańos: "Un político con propuestas vanguardistas para la educación peruana" (D9); "Participar en una organización de desarrollo docente (sindical o profesional) (D11); "También, me interesa incursionar en política, ocupando el cargo de congresista para velar por los intereses del magisterio peruano. Postulando como independiente" (D19).

A partir de las respuestas se pueden apreciar tres tipos de itinerarios proyectados según el rango de escenarios. El más característico es el de perspectivas múltiples, presente en once de los docentes y que se define por incluir un mínimo de cuatro hasta seis de los ocho escenarios en sus próximos diez ańos. Por ejemplo, encontramos un docente que se visualiza logrando metas en el escenario de la gestión educativa, de mejoras en su escuela actual, continuando sus estudios, enseñando en la universidad, publicando y logrando ascender en su carrera. Otro itinerario es el de perspectivas restrictivas, que representa a ocho docentes que se proyectan entre dos o tres escenarios posibles. Por último, se encuentran los que hemos tipificado como perspectivas centradas, para dar cuenta de la visión de un docente centrado en un escenario predominante relacionado con el de los estudios, siendo un caso muy ambicioso al proponerse durante los siguientes diez años estudiar tres doctorados, otra maestría y concluir otra carrera.

\section{Consideraciones Finales}

Este programa de beca, como parte de una política de inversión en el talento humano y de revalorización del docente peruano, ha marcado un hito en la historia de la política de formación continua del docente de educación básica del sector público. Consideramos como rasgos positivos por consolidar del programa: la oferta de beca integral con dedicación a tiempo completo; la cobertura descentralizada que beneficia a docentes no solo de la capital; con acceso para ambos géneros, el carácter meritocrático para su postulación, abierto a docentes de los diferentes niveles de la educación básica y orientado a temáticas propias de la educación básica.

Este estudio propone una discusión desde la perspectiva subjetiva sobre la necesidad de reinterpretar los múltiples impactos de las políticas de formación continua desde las percepciones de los docentes y sus capacidades de agenciamiento, como experiencia de autodeterminación y autonomía, como ha sido abordado conceptualmente en el segundo punto de este articulo. El análisis de los cuatro tipos de dilemas que subyacen en su decisión de postular a la 
beca de posgrado - maestría versus familia; maestría versus escuela; maestría versus otro estudio; maestría versus no migrar- evidencian la movilización subjetiva no solo de los docentes sino también de sus entornos (sentimientos en conflicto por dejar a sus familiares y alumnos y, a la vez, de expectativa por los estudios) y el desarrollo de sus competencias biográficas (aprender a decidir sobre la propia vida), así como, de resiliencia para adaptarse y enfrentar los riesgos (Beltamn, Mansfield \& Price, 2011) de un contexto nuevo marcado por las exigencias académicas y de una nueva ciudad como es la capital. Una implicancia a nivel de política institucional de este punto es la necesidad de un nivel de asesoramiento mayor para abordar de mejor manera las consecuencias subjetivas de estos dilemas que puedan perjudicar los estudios y equilibrio emocional de los docentes.

Pero el impacto sobre la subjetividad del docente no solo es hacia atrás, en el momento de resolver el dilema de postular o no a la maestría, sino también puede haber impactado en su proyección profesional. Probablemente el efecto de la maestría haya diversificado más su rango de escenarios donde visualizarse en los próximos diez años y donde no necesariamente se tratan de escenarios excluyentes, sino que podrían en ciertos casos ser simultáneos o con secuencias no siempre lineales. Esa diversificación de ocho escenarios que los resultados muestran, puede implicar un reimpulso hacia una visión más flexible de la carrera y el construir un imaginario profesional más nutrido de opciones y no solo centrado en la docencia en aula, aportándole así de recursos subjetivos para su experiencia profesional de agenciamiento.

Ese abanico de opciones coincide con los resultados de la Encuesta Nacional a Docentes de Instituciones Educativa Públicas y Privadas del Perú, en la que $56 \%$ de los encuestados expresan como sus perspectivas laborales para dentro de cinco años el acceder a cargos de gestión, de especialistas o de formadores de docentes (Minedu-CNE, 2015), el 33\% seguir como docente y $11 \%$ dejar la carrera. En nuestro estudio, salvo el que se visualiza postulando como parlamentario, el resto se proyecta en diversos escenarios relacionados al campo profesional educativo. Una de las restricciones para esa movilización en determinados escenarios es que los docentes becarios han asumido como compromiso con Pronabec de retornar a su escuela por un periodo no menor al doble del tiempo de los ańos de estudios becados.

Expresamos una limitación del estudio para determinar con mayor certeza los cambios en sus orientaciones hacia el futuro que la experiencia de la maestría haya producido. Un estudio más longitudinal de los docentes becarios podría ofrecernos más luces sobre la evolución de sus perspectivas profesionales y sus concreciones. 
Una de las implicancias críticas a nivel de política docente es la poca articulación entre una de las cuatro áreas de desempeño laboral que contempla la ley de reforma magisterial del Perú (Minedu, 2013), como es la de Innovación e Investigación, y los escenarios de futuro laboral que los docentes analizados plantean; mayormente no mencionan el postular a dicha área, siendo una élite que estaría en condiciones para desempeñarse en esa función.

En este estudio queremos enfatizar en la importancia de integrar otras miradas sobre las políticas de la formación continua en contraste con enfoques que solo miden la cobertura de dichas políticas o su incidencia en el rendimiento académico de los alumnos. Creemos que estas últimas, ayudan a valorar la dimensión objetiva de los impactos, pero que requieren complementarse con la dimensión subjetiva, donde las motivaciones, las expectativas y la emocionalidad juegan un rol en el docente desde el inicio de sus experiencias de formación continua.

\section{REFERENCIAS BIBLIOGRÁFICAS}

Alcaldía Mayor de Bogotá (2012). Plan de Desarrollo 2012-2016. Bogotá Humana. Recuperado de http://www.sdp.gov.co/portal/page/portal/PortalSDP/ ciudadania/PlanesDesarrollo/BogotaHumana/2012_2016_Bogota_ Humana_Plan_Acuerdo489_2012.pdf

Andrade, D. \& Fraga, L. (2010). Pesquisa trabalho docente na educação básica no Brasil. Grupo de Estudos Sobre Política Educacional e Trabalho Docente GESTRADO/UFMG.

Beltamn, S.,Mansfield, C. \& Price, A. (2011). Thriving not just surviving: A review of research on teacher resilience. Educational Research Review, 6(3), 185-207. https://doi.org/10.1016/j.edurev.2011.09.001

Biesta, G. \& Tedder, M. (2007). Agency and learning in the lifecourse: Towards an ecological perspective. Studies in the Education of Adults, 39(2), 132149. https://doi.org/10.1080/02660830.2007.11661545

Cherry, N. (2014). New stories of mastery: constructive learning in the face of complex dilemmas of practice. Studies in Continuing Education, 36(3), 241-256. https://doi.org/10.1080/0158037X.2014.904781

Cochran, C., Carter, G. \& Dorsey, D. (2003). Identifying Career Paths: A Review of the Literature. Technical Report N 43. July, Minnesota: Personnel Decisions Research Institute.

Comisión Nacional de investigación Científica y Tecnológica (Conicyt) (2013). Becas de magíster en el extranjero para profesionales de la educación becas 
Chile - convocatoria 2013. Recuperado de http://www.conicyt.cl/becasconicyt/2013/05/magister-extranjero-profesionales-educacion-2013/

Comisión Nacional de investigación Científica y Tecnológica (CONICYT) (2014). Becas de magíster en Chile para profesionales de la educación. Año Académico 2014-2015. Recuperado de http://www.conicyt.cl/becasconicyt/files/2014/07/RESUMEN-DE-FALLO.pdf

Cuenca, R. \& Stojnic. L. (2008). La cuestión docente Perú: Carrera Pública magisterial y el discurso del desarrollo profesional. Lima, Perú: Flape.

Darling-Hammond, L. (2000). Teacher quality and student achievement: a review of state policy evidence. Education Policy Analysis Archives, 8(1). https:// doi.org/10.14507/epaa.v8n1.2000

Eckert, J., Ulmer, J., Khachatryan, E. \& Ledesma, P. (2016). Career pathways of teacher leaders in the United States: adding and path-finding new professional roles. Professional Development in Education, 42(5), 687-709. https://doi.org/10.1080/19415257.2015.1084644

El Peruano (3 de noviembre de 2014). S/. 30 mllns. en becas para maestro. Recuperado de http://www.elperuano.pe/noticia-s-30-mllns-becas-paramaestros-22619.aspx

Eteläpelto, E., Vähäsantanen, K. \& Hökkä, P. (2015). How do novice teachers in Finland perceive their professional agency? Teachers and Teaching, 21(6), 660-680. https://doi.org/10.1080/13540602.2015.1044327

Foreque, F. (11 de mayo de 2015). MEC quer acelerar expansão de pós entre docentes da educaçáo básica. Folha de S. Paulo. Recuperado de http:// www1.folha.uol.com.br/educacao/2015/05/1627427-mec-quer-acelerarexpansao-de-pos-entre-docentes-da-educacao-basica.shtml

Fransson, G. \& Grannäs, J. (2013). Dilemmatic Spaces in Teachers Work Towards a Conceptual Framework for Dilemmas in Teachers Work. Teachers and Teaching Theory and Practice, 19(1), 4-17. https://doi.org/10. 1080/13540602.2013.744195

Gandhi, G. (2006). Teacher characteristics and student performance in India: A pupil fixed effects approach. Global Poverty Research Group-University of Oxford.

Gusmão, R. (2010). Desarrollo de las capacidades de investigación en ciencias sociales en Brasil. En CICS, Informe Mundial sobre las Ciencias Sociales (pp. 132-136). París: Unesco.

Harris, D. \& Sass, T. (2007). Teacher Training, Teacher Quality, and Student Achievement. CALDER Center. https://doi.org/10.1037/e722772011-001

Ingersoll, R. (2011). Estudio comparado sobre la formación y antecedentes académicos de los docentes en seis naciones. PREAL Doc. N 56. 
Ingersoll, R., Merrill, L. \& May, H. (2014). What are the effects of teacher education and preparation on beginning teacher attrition? Philadelphia: CPRE, University of Pennsylvania.

Instituto Nacional de Estadística e Informática (INEI) (2014). Resultados de la Encuesta Nacional a Instituciones Educativas de Nivel Inicial, Primaria y Secundaria, 2013. Lima, Perú: INEI

Johnston-Anderson, N. (2016). Teacher career trajectories and aspirations in context: A mixed methods study of second-stage teachers in New South Wales. (Doctoral Dissertation, The University of Sidney).

Kelchtermans, G. (1993). Getting the story, understanding the lives: from career stories to teachers' professional development. Teaching \& Teacher Education, 9(5-6), 443-456. https://doi.org/10.1016/0742-051X(93)90029-G

Latifoglu, A. (2016). Staying or Leaving? An Analysis of Career Trajectories of Beginning Teachers. International Studies in Educational Administration, 44(1), 55-70.

Martínez, P. (2006). Perspectiva futura del Perú y el nivel de satisfacción con él. Liberabit, 12, 113-121.

Mineducación-Colombia (2016). Gina Parody lanza segunda convocatoria del Programa de Becas para Docentes que busca beneficiar a 3.566 profesores de colegios oficiales de todo el país. Recuperado de http://www.mineducacion.gov.co/1759/w3-printer-356839.html

Ministerio de Educación-Consejo Nacional de Educación (MINEDU-CNE) (2015). Encuesta Nacional a Docentes de Instituciones Educativa Públicas y Privadas del Perú. Recuperado de https://issuu.com/ ministerioeducacionperu/docs/encuesta_nacional_a_docentes

Ministerio de Educación-Perú (Minedu) (2013). Reglamento de la Ley No 29944, Ley de Reforma Magisterial. Decreto Supremo No 004-2013-ED.

Ministerio de Educación-Perú (Minedu) (2014). Magnitudes de la educación en el Perú. Número de docentes en el sistema educativo por tipo de gestión. http://escale.minedu.gob.pe/magnitudes-portlet/reporte/cuadro?anio=19 \&cuadro=309\&forma=U\&tipo_ambito=ambito-ubigeo

Ministerio de Educación-Perú-Programa Nacional de Becas y Créditos Educativos (Minedu-Pronabec) (2014). Resolución Jefatural N 065-2014, 7 agosto.

Minner, S. \& Beane, A. (1983). Professional Dilemmas for Teachers of Mentally Retarded. Children Education and Training of the Mentally Retarded, 18(2), 131- 133.

Organisation for Economic Co-operation and Development (OECD) (2014). Talis 2013 Results: An International Perspective on Teaching and Learning. París, Francia: OECD. 
Pittman, G. (2000). Dilemmatic constructions of career in the career counseling interview. The Career Development Quarterly, 48, 226-236. https://doi. org/10.1002/j.2161-0045.2000.tb00288.x

Programa Nacional de Becas y Crédito Educativo (Pronabec) (2014). Memoria Institucional 2012-2014. Lima: Pronabec.

Programa Nacional de Becas y Crédito Educativo (Pronabec) (2015). Memoria Institucional 2012-2015. Lima: Pronabec.

Quadrado, L. \& Rocha-de-Oliveira, S. (2015). História de Vida e Trajetórias Profissionais: Estudo com Executivos Brasileiros. Revista de Administração Contemporânea, 19(4), 525-543. https://doi. org/10.1590/1982-7849rac20151951

Rama, C. (2006). Los postgrados en América Latina en la sociedad del saber. En IESALC, Informe sobre la Educación Superior en América Latina y el Caribe. 2000-2005. La metamorfosis de la educación superior (pp. 43-55). Venezuela: IESALC.

Red Académica Bogotá (s.f.). Posgrados. Recuperado de http://www.redacademica.edu.co/es/formaci\%C3\%B3n-de-docentes/formaci\%C3\%B3n/ postgrados-docentes.html

Rinke, C. (2008). Understanding teachers' careers: Linking professional life to professional path. Educational Research Review, 3, 1-13. https://doi. org/10.1016/j.edurev.2007.10.001

Sahlberg, P. (2010). The Secret to Finland's Success: Educating Teachers. Research Brief. Stanford Center for Opportunity Policy in Education.

Sánchez, G. (2006). De la capacitación hacia la formación continua de los docentes aportes a la política (1995-2005). Lima: Minedu.

Santos, P. (2004). Career Dilemmas in Career Counseling Groups: Theoretical and Practical Issues. Journal of Career Development, 31(1), 31-44. https:// doi.org/10.1177/089484530403100103

Schön, D. (1992). La formación de los profesionales reflexivos. España: MEC-Paidós.

Secretaria da Educação do Estado de São Paulo (2015). Programa Mestrado \& Doutorado. Recuperado de http://www.escoladeformacao.sp.gov.br/portais/Default.aspx?tabid=3305

Sheng-nan, L. \& Da-ming, F. (2015). How culture matters in educational borrowing? Chinese teachers' dilemmas in a global era. Cogent Education, 2, 1-15.

Sime, L. (2008). Los dilemas profesionales: una aproximación al estudio de las trayectorias de los educadores. Actas del V Encuentro Internacional Kipus: Las transformaciones de la profesión docente frente a los actuales desafíos, Lima. Recuperado de http://www.redkipusperu.org/files/86.pdf 
Strahan, D. (2016). Mid-career teachers' perceptions of self-guided professional growth: strengthening a sense of agency through collaboration. Teacher Development, 20(5), 667-681. https://doi.org/10.1080/13664530.2016.1 190782

Tharenou, P. (2015). Chinese international business graduates: A career dilemma: Repatriate or stay? Journal of Management \& Organization, 21(1), 37-59. https://doi.org/10.1017/jmo.2014.68

The MetLife (2013). Survey of the American Teacher: Challenges for School Leadership. https:/www.metlife.com/assets/cao/foundation/MetLife-TeacherSurvey-2012.pdf

Tobón, D., Valencia, G., Ríos, P. \& Fredy, J. (2008). Organización jerárquica y logro escolar en Medellín: un análisis a partir de la función de producción educativa. Lecturas de Economía, 68,145-173.

van Rijswijk, M., Akkerman, S., Schaap, H. \& van Tartwijk, J. (2016). Past perceptions and future expectations: Sensed dis/continuity at the start of teacher education. Teaching and Teacher Education, 58, 99-108. https://doi. org/10.1016/j.tate.2016.05.002

Zavala, M. A. y Castañeda, S. (2014). Fenomenología de agencia y educación. Notas para el análisis del concepto de agencia humana y sus proyecciones en el ámbito educativo. Magister, 26, 98-104. https://doi.org/10.1016/ S0212-6796(14)70024-6 